\title{
Availability and feasibility of structured, routine collection of comorbidity data in a colorectal cancer multi-disciplinary team (MDT) setting
}

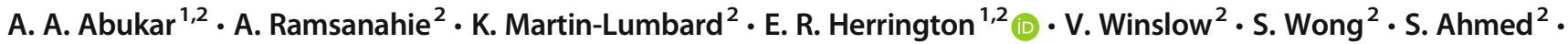 \\ M. A. Thaha ${ }^{1,2}$
}

Accepted: 24 April 2018 / Published online: 3 May 2018

(C) The Author(s) 2018

\begin{abstract}
Purpose Availability of comorbidity assessment at multi-disciplinary team (MDT) discussions is cornerstone in making the MDT process more robust and decisive in optimising treatment and improving quality of survivorship. Comorbidity assessments using tools, such as the ACE-27 questionnaire would aid in optimising the decision-making process at MDTs so that treatment decisions can be made without delay. This study determined the availability of comorbidity data in a CRC MDT and the feasibility of routine comorbidity data collection using the validated ACE-27 questionnaire. Secondary aims determined the optimal time and method of collecting comorbidity data.

Methods A retrospective mapping exercise (phase I; 6-months) examined the availability of comorbidity data within the MDT. Phase II prospectively collected comorbidity data using ACE-27 for a 3-month period following a short pilot.

Results In phase I, 73/135 (54\%) patients had comorbidity data readily available informing the MDT discussion; 62 patients lacked this information. After a review of the patient records, it was clear that 41 of these 62 also had comorbidities and 21 out of the $135 \mathrm{had} \geq 2$ major system disorders. Common referral sources to the MDT were surgical outpatient clinics (42\%) and the endoscopy unit (13\%). The average lead-time from referral to MDT discussion was 14 days. In phase II, an ACE-27 questionnaire was prospectively administered in 50 patients, mean age 54 years (range 20-84). Male: female ratio 26:24. Average time to administer ACE-27 was 4.8 min (range 1-15).

Conclusions The phase I study confirmed the widely acknowledged view of poor comorbidity data availability within a CRC MDT. Phase II demonstrated the feasibility of routinely collecting comorbidity data using ACE- 27.
\end{abstract}

Keywords Colorectal cancer $\cdot$ MDT $\cdot$ Comorbidity $\cdot$ ACE-27

What does this paper add to the literature? This is the first study in the literature to assess the availability of comorbidity data in a CRC MDT and to prospectively assess the feasibility of using ACE-27 comorbidity index in a colorectal cancer setting.

M. A. Thaha

m.a.thaha@qmul.ac.uk

1 Blizard Institute, National Bowel Research Centre, Barts and The London School of Medicine and Dentistry, Queen Mary University London, London, UK

2 Department of Colorectal Surgery, Surgery \& Cancer CAG, The Royal London Hospital, Barts Health NHS Trust, London, UK

\section{Introduction}

Comorbidities Colorectal cancer (CRC) is the second most common cause of cancer death in the UK and the fourth most common type of cancer in men and women. More than 41,000 new cases of CRC were diagnosed in 2014, with an incidence rate of approximately 64 CRC patients per 100,000 individuals $[1,2]$. With an ageing population, the prevalence of comorbidities is set to increase [3, 4]. The presence of comorbid conditions affects treatment pathways and prognosis of patients [5]. Comorbidity reduces the quality of life, may complicate major surgical procedures and increases the risk of hospitalisation and mortality [3, 4]. This highlights the need for comorbidity measurements from a clinical point of view as 
they may help healthcare providers to respond effectively to the overall severity and healthcare needs of the individual, improving the quality of survivorship. Multi-disciplinary team (MDT) meetings are the gold standard in the management of cancer patients in the UK and are similar to tumour boards in other national settings.

MDT meetings Currently, all patients diagnosed with or suspected to have cancer are discussed at MDT meetings. MDT meetings usually take place once weekly bringing together healthcare professionals with an array of skills and expertise. They aim to ensure high-quality decision-making with regard to the diagnosis, treatment and aftercare of patients and they are associated with an improved 5-year survival in colorectal cancer [6, 7]. A number of patient-based factors are discussed at MDTs, such as frailty, patient preference, psychological and social needs; comorbidities are an important part of a holistic assessment. There are increasing pressures on MDTs in the UK, which mean that timely and accurate data are increasingly important, particularly in respect of cancer [8]. Two studies assessing MDT decision-making in an upper GI and CRC setting revealed that MDT decisions were not being implemented primarily due to a lack of information concerning patients' comorbidities $[9,10]$. Lack of relevant information on the burden of comorbidities and overall fitness of the patient may deter an MDT from making holistic decisions about the patient's care and may necessitate repeated discussions at the MDT.

ACE-27 The ACE-27 index was derived by Piccirillo and colleagues from the Modified Medical Comorbidity instrument which was itself derived from the Kaplan Feinstein Index (KFI). The ACE-27 is an example of organ/system-based approach; this methodology measures how comorbidity impacts on the functions of the bodily system, e.g. cardiovascular, gastrointestinal, respiratory etc. The aim of the ACE-27 development was to assess specifically for comorbidity in patients with cancer and has been utilised in a number of studies of which cancer was the context. Based on experience from research and clinical judgement, 27 conditions were identified and included into the index. The ACE-27 comorbidity index, similar to the KFI, grades the selected 27 comorbid conditions into 3 grades of severity from which they are summarised to give an overall rating. Specifically, comorbid diseases are classified separately as mild, moderate and severe in relation to the extent of organ decompensation and prognostic impact. These individual grades are then used to assign patients to the overall ACE-27 score, according to the highest ranked comorbidity. If patients have more than two moderate diseases in different organs an overall score of severe is assigned $[3,11]$. It is one of several different comorbidity assessment tools and has been tested for validity and reliability in a number of studies; it can be completed by patients or by clinicians (see discussion below).
Aims In this study, we primarily aimed to determine the availability of comorbidity data in a CRC MDT and assessed the feasibility of routine comorbidity data collection using the validated ACE-27 questionnaire. Secondary aims of the study included determining the optimal time and method of comorbidity data collection.

\section{Methods}

Population The study was conducted in an inner London CRC MDT serving a multi-ethnic, predominantly deprived population. The population was chosen given the comparatively high rate of colorectal cancer as against the UK average and the location of the research team. The London Borough of Tower Hamlets located in the eastern part of London has a population of 254,096, with one of the highest ethnic minority populations in the capital. Half of the borough's population are from Asian or Asian British, Black or Black British or other ethnic background. Of these ethnic groups, Bangladeshis (32\%) represent the largest ethnic group [12].

Consent and ethics The research team were drawn from the clinical team and had access to patient data. All patients gave appropriate consent for their data to be used in the study, which received relevant ethics approval.

Context We conducted this pilot study to assess availability of comorbidity data at CRC MDT and to find out the feasibility of using the validated ACE-27 questionnaire to capture this data. The NHS working party on comorbidity assessment in cancer report in 2001 recommended the use of the ACE-27 [13]. In 2010, after the 2009 national workshop on 'Comorbidity in Cancer,' the National Cancer Intelligence Network (NCIN) sought applications from MDT's in England for pilot projects on collecting ACE-27 scores as a routine comorbidity measurement for use in MDT's. The colorectal cancer MDT at The Royal London Hospital was recruited to evaluate the feasibility of collecting ACE-27 comorbidity scores in the context of CRC MDT.

Phase I In phase I, a retrospective mapping exercise was carried out during a 6-month period (January 2012 to June 2012) to assess the accuracy of the recorded patients' comorbidities data during MDT meetings. Data pertaining to patients' comorbidity status were collected from CRC MDT meeting records (historic cohort). Detailed evaluation of outpatient clinic letters, electronic patient records and general practitioner referral letters were carried out for all the patients in order to compare the completeness of comorbidity data available at the CRC MDT meeting and to delineate optimal times for data collection. 
Phase II In phase II, comorbidity data were prospectively collected over a 3-month period using the validated ACE-27 comorbidity index. The ACE-27 was administered by the surgical team (consultant, specialty, core and foundation trainees) in surgical outpatient clinics, surgical ward and preassessment clinic to assess both the feasibility of routinely administering the ACE 27 forms and measuring comorbidity in CRC patients. This phase II firstly involved organising and appropriately training three foundation year 1 doctors as part of a team (juniors doctors within 1 year of qualifying) to conduct the ACE 27 questionnaire on 11 patients in two different settings. These settings were the (1) inpatient ward and (2) pre-assessment clinic.

Review A review of the process was then conducted following this period, to further train remaining members of the team and to resolve some of the discrepancies that occurred in the interpretation of the ACE-27 form and its use. Subsequently, the ACE- 27 forms were completed prospectively in 50 colorectal patients.

\section{Results}

Phase I In phase I, there were 135 patients who were newly referred to the CRC MDT meeting for first discussion. Of the 135 patients, $73(54 \%)$ patients had comorbidity data available informing the CRC MDT meeting discussion. In the 62 patients $(46 \%)$ with no comorbidity data available at MDT meeting, a detailed assessment of patient records revealed 41 (30\%) patients to have comorbidities including $\geq 2$ major system disorders in 21 (16\%) patients (Fig. 1).

Of the 135 patients reviewed by the MDT, only 100 patients had available data to identify how quickly patients were discussed from time of referral to first discussion at the MDT meeting. This revealed that the majority $(43 \%)$ of patients

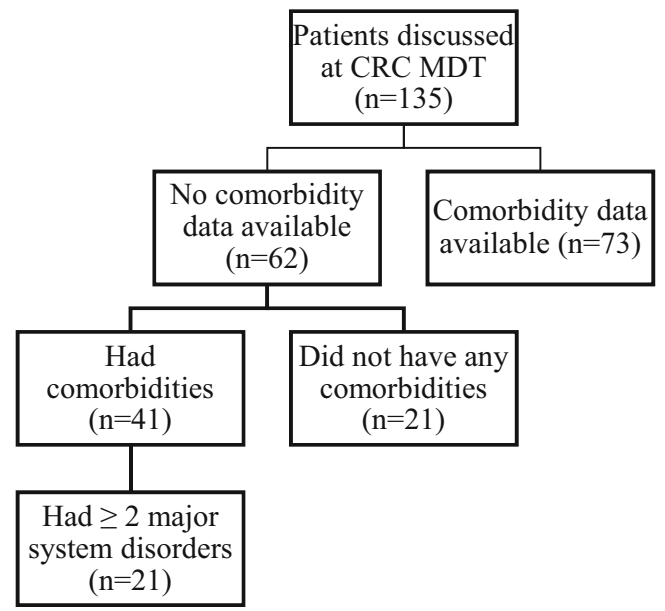

Fig. 1 Flow chart summarising availability of comorbidity data at CRC MDT were discussed within 1 week of referral to the MDT meeting. However, the average lead time to discussion from referral to the CRC MDT meeting was 14 days (range 1 to 64 days). When the sources of referral for the 135 patients were mapped, it showed that common sources of patient referral to the MDT were surgical outpatient clinics (42\%), emergency on-call take $(18 \%)$ and the endoscopy (13\%) department (Table 1).

Phase II In phase II, ACE-27 was administered in 50-patients in surgical outpatient clinics, surgical ward and pre-assessment clinic setting. The mean age was 54 years (range 20-84 years). Out of the 50 patients, 26 were male, while 24 were female. The average time to administer the ACE-27 was 4.8 min (range 1$15 \mathrm{~min}$ ). In the grading of the ACE-27, 17 patients (34\%) were grade 3,11 patients $(22 \%)$ were grade 2,11 patients $(22 \%)$ were grade 1 and 11 patients (22\%) were grade 0 (Fig. 2).

\section{Findings}

Phase I of the study found documented evidence of comorbidity data in $54 \%$ of patients only. Significantly, a further $16 \%$ of patients had comorbidities affecting $\geq 2$ major systems. This knowledge would have been critical in the MDT's decision-making. The study found that a structured approach of prospectively collecting comorbidity data, preferably using a validated questionnaire, would increase the availability of such data: in phase II, $100 \%$ of patients had comorbidity data. The ACE-27 questionnaire was easy to use, and produced consistent data for the MDT to interpret. As a result, the structured collation of comorbidity data has since been integrated into the MDT information process.

\section{Discussion}

Comorbidities Comorbidities refer to diseases that coexist with the disease of interest. They are one of many patient data that are discussed at MDTs, such as patient preference, acceptability, frailty and psychological needs. The presence of comorbidities may alter treatment choice and correlates with survival and complications. The treatment of CRC can include

Table 1 Sources of referral to MDT meetings

\begin{tabular}{ll}
\hline Source of referral & Number of patients \\
\hline Emergency & 24 \\
Endoscopy & 17 \\
Surgical outpatients & 57 \\
In-hospital specialty & A \\
Tertiary & 27 \\
\hline
\end{tabular}

${ }^{\mathrm{A}}$ General medicine, hepatopancreaticobiliary (HPB), urology etc. 


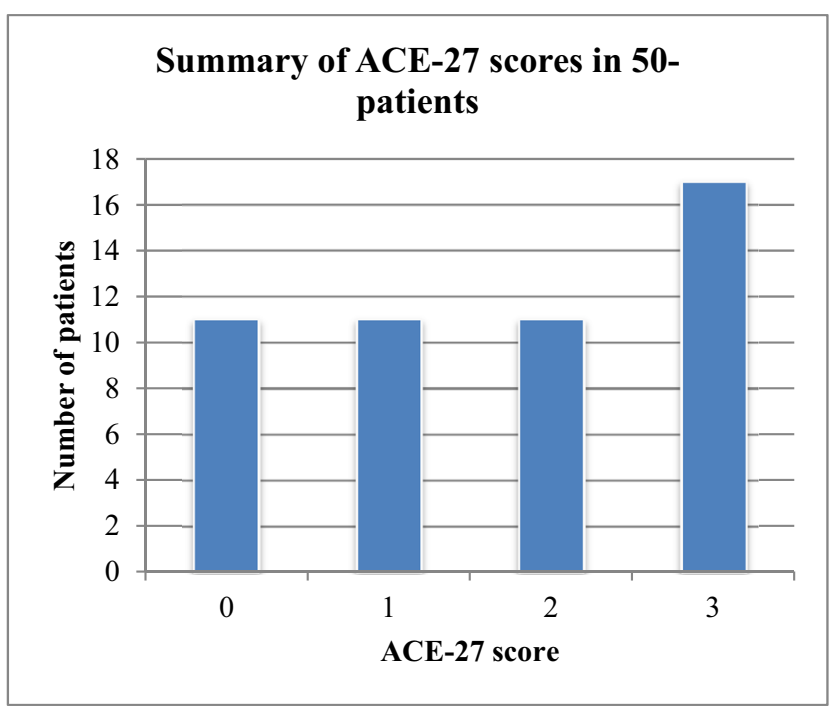

Fig. 2 ACE-27 scores in 50-patients

extensive surgery, chemotherapy and radiotherapy; hence, such patients are, potentially, at higher risk of adverse outcomes. Decision-making at the MDT meeting is influenced by expertise, facility and resource factors, which would be similar for all patients discussed. However, the variable of patient factors changes with each patient discussed. Blazeby and colleagues assessed the reasons for discordance between the MDT decision and the final treatment implemented in an upper gastrointestinal cancer MDT setting. The majority (43.9\%) were related to comorbid health issues [9]. Similarly, Wood and colleagues in a colorectal cancer MDT setting found the primary reason for an MDT decision not being implemented $(40 \%)$ related to comorbid health issues [10].

Cost benefits MDT meetings use up significant resources and there are almost 1500 teams in England. The cancer MDT meetings are estimated to cost the NHS in England $£ 50$ million a year for preparation and a similar amount for attendance. Having real-time information about comorbidity would aid in making the MDT process more robust and decisive in optimising treatment, improving quality of survivorship and being cost-effective [8].

Retrospective data collection Phase I of the study confirmed the previously widely acknowledged view of poor comorbidity data availability within a CRC MDT discussion. Our study has shown on average there were 14 days from referral to discussion at the CRC MDT; hence, sufficient time exists to capture comorbidity data prior to discussion. Additionally, the mapping exercise revealed potential locations to administer the ACE-27 questionnaire, which includes surgical outpatient clinics, emergency department and at endoscopy.

Prospective data collection We initially planned to send the ACE-27 questionnaire to patients to fill out prior to their attending surgical outpatients or pre-assessment clinic; however, we judged that patients might struggle with the medical terminology, which precluded its use in patient questionnaires. Furthermore, this would have been inappropriate for our local population as English is not the first language for a significant majority. Instead, the ACE-27 was administered by junior and senior clinicians following a short 10-min training and on average took $4.8 \mathrm{~min}$ to complete prospectively. Training was embedded into the process of induction for all new members of staff as well as refresher training for existing members of staff; it was felt to be an extremely important and welcome part of discussing patients' needs holistically. Roger et al.'s study used the ACE- 27 index retrospectively and took on average of $10 \mathrm{~min}$ to complete [11]. They encountered difficulties in administering the ACE-27 retrospectively as many of the items listed in the index were not mentioned in medical notes and some of the mentioned comorbidities lacked sufficient detail about their severity to enable accurate scoring. Our study also found that using the ACE- 27 index prospectively would be more feasible routinely rather than retrospectively.

Future studies Other comorbidity indices used in colorectal cancer reported in the literature include the Charlson comorbidity index (CCI), Elixhauser comorbidity index (ECI) and the National Institute on Ageing/National Cancer Institute Collaborative Study on Comorbidity and Cancer Index (NIA/NCI). CCI is an example of a weighted index, which consists of a number scoring system based on 19 weighted conditions that can subsequently be used for comparison and evaluation of risk. The ECI is an example of counts of individual disease and is a more recent risk-adjustment model comprising 31 conditions including weight loss and obesity. The NIA/NCI is another example of using counts of individual disease and was the only index designed exclusively utilising cancer patients in the NCI's SEER program. The ELI and NIA/NCI evaluate the comorbidity burden only through a quantitative point of view, whereas the ACE-27 and $\mathrm{CCI}$ explore disease severity weighting the comorbid diseases on clinical impact. Studies in the literature found the aforementioned indices all have a good utility in predicting CRC patient's survival [3, 14]. Future studies comparing the feasibility of collecting comorbidity data using the three indices against ACE 27 in CRC MDT setting are needed. We conducted our study in a specific national setting and local population; it would be valuable to test these findings in different settings and on a larger scale.

\section{Conclusions}

Our study highlights the importance of having available a tool to collect comorbidity data prior to a CRC MDT discussion. Some limitations in the use of ACE-27 exist: different 
laboratory value units to those used in the UK and important comorbidities are omitted, which necessitate modifications [3, 11]. Further studies assessing the impact of ACE-27 comorbidity scores on the CRC MDT's decision are required.

Funding The authors would like to thank the National Cancer Intelligence Network (NCIN) for their kind funding and for obtaining the necessary licencing agreement from the developers of ACE-27 questionnaire for its use in the study.

\section{Compliance with ethical standards}

Conflict of interest The authors declare that they have no conflicts of interest.

Informed consent Any necessary informed consent was obtained from all individual participants included in the study.

Open Access This article is distributed under the terms of the Creative Commons Attribution 4.0 International License (http:// creativecommons.org/licenses/by/4.0/), which permits unrestricted use, distribution, and reproduction in any medium, provided you give appropriate credit to the original author(s) and the source, provide a link to the Creative Commons license, and indicate if changes were made.

\section{References}

1. Craigie AM, Caswell S, Paterson C, Treweek S, Belch JJF, Daly F, Rodger J, Thompson J, Kirk A, Ludbrook A, Stead M, Wardle J, Steele RJC, Anderson AS (2011) Study protocol for BeWEL: the impact of a Body WEight and physicaL activity intervention on adults at risk of developing colorectal adenomas. BMC Public Health 11:1-8

2. Cancer Research UK (2017) Bowel (colorectal) cancer-UK incidence statistics. [online] Available at: < http://www.cancerresearchuk. org/health-professional/cancer-statistics/statistics-by-cancer-type/ bowel-cancer/incidence\#heading-Zero. Accessed 22 April 2017

3. Sarfati D (2012) Review of methods used to measure comorbidity in cancer populations: no gold standard exists. J Clin Epidemiol 65: 924-933

4. Janssen-Heijnen MLG, Maas HAAM, Houterman S, Lemmens VEPP, Rutten HJT, Coebergh JWW (2007) Comorbidity in older surgical cancer patients: influence on patient care and outcome. Eur J Cancer 43:2179-2193

5. Dekker JWT, Gooiker GA, van der Geest LGM, Kolfschoten NE, Struikmans H, Putter H, Wouters MWJM, Tollenaar RAEM (2012) Use of different comorbidity scores for risk-adjustment in the evaluation of quality of colorectal cancer surgery: does it matter? Eur J Surg Oncol 38:1071-1078

6. National Cancer Action Team (2010) The characteristics of an effective multidisciwplinary team (MDT). Available at: http:// webarchive.nationalarchives.gov.uk/20160805125148/http://www. nhsiq.nhs.uk/media/2444560/ncatmdtcharacteristics.pdf. Accessed 22 April 2017

7. Cancer Research UK (2017) Meeting patients' needs: improving the effectiveness of multidisciplinary team meetings in cancer services. Available at http://www.cancerresearchuk.org/sites/default/ files/full_report_meeting_patients_needs_improving_the effectiveness_of_multidisciplinary_team_meetings_.pdf. Accessed 22 April $201 \overline{7}$

8. Taylor C, Munro A, Glynne-Jones R, Griffith C, Trevatt P, Richards M, Ramirez AJ (2010) Multidisciplinary team working in cancer: what is the evidence? BMJ 340:951

9. Blazeby JM, Wilson L, Metcalfe C, Nicklin J, English R, Donovan JL (2006) Analysis of clinical decision-making in multidisciplinary cancer teams. Ann Oncol 17:457-460

10. Wood JJ, Metcalfe C, Paes A, Sylvester P, Durdey P, Thomas MG, Blazeby JM (2008) An evaluation of treatment decisions at a colorectal cancer multi-disciplinary team. Color Dis 10: 769-772

11. Rogers SN, Aziz A, Lowe D, Husband DJ (2006) Feasibility study of the retrospective use of the Adult Comorbidity Evaluation index (ACE-27) in patients with cancer of the head and neck who had radiotherapy. Br J Oral Maxillofacial Surg 44:283-288

12. London Borough of Tower Hamlets (2013) Ethnicity in Tower Hamlets: analysis of 2011 Census data. Available at http://www. towerhamlets.gov.uk/Documents/Borough_statistics/Ward profiles/Census-2011/RB-Census2011-Ethnicity-2013-01.pdf. Accessed 22 April 2017

13. Kastner C, Armitage J, Kimble A, Rawal J, Carter PG, Venn S (2006) The Charlson comorbidity score: a superior comorbidity assessment tool for the prostate cancer multidisciplinary meeting. Prostate Cancer Prostatic Dis 9:270-274

14. Marventano S, Grosso G, Mistretta A, Bogusz-Czerniewicz M, Ferranti R, Nolfo F, Giorgianni G, Rametta S, Drago F, Basile F, Biondi A (2014) Evaluation of four comorbidity indices and Charlson comorbidity index adjustment for colorectal cancer patients. Int J Color Dis 29:1159-1169 